\title{
Analysis of Correlation on Performance of Golden Code Based on HF Watterson Model
}

\author{
Xin Wang ${ }^{1}$, Lijia Ge ${ }^{2}$
}

\author{
${ }^{1}$ Chongqing University of Posts and Telecommunications,Chongqing, 400065, China ${ }^{2}$ Chongqing \\ University of Posts and Telecommunications, Chongqing, 400065, China
}

Keywords: HF communication, Space-Time Coding, Golden Code, correlation channel

\begin{abstract}
HF communication is one of the primary means of long distance communication. In recent years, because of its small transmission power and strong ability of anti-interference, it is widely used in various communication fields. The space-time coding has also been extensively studied to increase the channel capacity of effective technology. The Golden Code, a full rate and full diversity code for the $2 \times 2$ coherent multiple-input multiple-output(MIMO) systems. This outperforms all previous know codes. This paper proposes an idea of which Golden Code is applied to HF communication. However a basic assumption in studying Golden Code is that the channels between different transmit antenna and different receive antenna pairs are independent, but it can not always hold in real-life. This paper introduces the system model about Golden Code. An expression for Golden Code is derived under correlation channel based on Watterson model. Finally, the analytical results are verified through our computer simulation for Golden Code.
\end{abstract}

\section{Introduction}

HF communication is important in wireless communication and applied widely for its low transmit power, establishing and maintenance cheaply, short establish period, device accomplished easily and simple operation.HF data rates are currently too low to support reliable video or other data-intensive communication because of low bandwidth allocations and challenging propagation conditions. The space-time coding as an important technology of MIMO has been successfully improve the performance of HF communication [1,2]. Space-time coding techniques can be divided into two categories, one is able to increase the system capacity of spatial multiplexing ; the other is designed to increase the link gain of Spatial diversity. The Golden Code for a $2 \times 2$ multiple-input multiple-output can achieve spatial multiplexing and Spatial diversity. This outperforms all previous know codes. We proposes an idea of which Golden Code is applied to HF communication.

However a basic assumption in studying Golden Code is that the channels between different transmit antenna and different receive antenna pairs are independent .We know in the actual propagation environment, because of the local distribution of local scatterers or limited distance between the antenna elements. Fading signals on different antennas behave in space may not be independent [3, 4].Analysis of correlation on performance of Golden code is necessary. This paper research the correlation on performance of Golden Code based on HF Watterson model.

\section{System model}

The Golden Code with two transmit antenna and two receive antenna can be modeled as shown in the figure 1. 


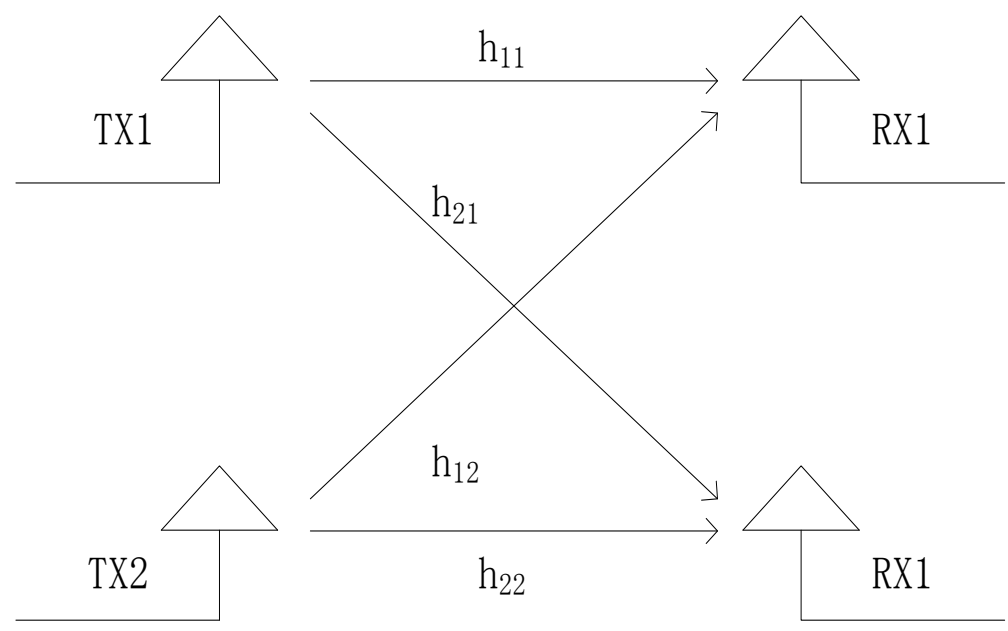

Fig.1 System model

In Fig.1 $h_{i j}$ is the channel from $i^{\text {th }}$ receive antenna to $j^{\text {th }}$ transmit antenna, $a+b \theta, \gamma(c+d \bar{\theta})$, $c+d \theta$ and $a+b \bar{\theta}$ are the transmitted symbols[5]. The received signal in the first time slot is:

$\left[\begin{array}{l}y_{1}^{1} \\ y_{2}^{1}\end{array}\right]=\left[\begin{array}{ll}h_{11} & h_{12} \\ h_{21} & h_{22}\end{array}\right]\left[\begin{array}{c}a+b \theta \\ \gamma(c+d \bar{\theta})\end{array}\right]+\left[\begin{array}{l}n_{1}^{1} \\ n_{2}^{1}\end{array}\right]$

Assuming that the channel remains constant for the second time slot, the received signal is in the second time slot is:

$\left[\begin{array}{l}y_{1}^{2} \\ y_{2}^{2}\end{array}\right]=\left[\begin{array}{ll}h_{11} & h_{12} \\ h_{21} & h_{22}\end{array}\right]\left[\begin{array}{l}c+d \theta \\ a+b \bar{\theta}\end{array}\right]+\left[\begin{array}{l}n_{1}^{2} \\ n_{2}^{2}\end{array}\right]$

where $\left[\begin{array}{l}y_{1}^{1} \\ y_{2}^{1}\end{array}\right]$ are t

are the received information at time slot 2 on receive antenna 1,2 respectively, $\left[\begin{array}{l}n_{1}^{1} \\ n_{2}^{1}\end{array}\right]$ are the noise at time slot 1 on receive antenna 1,2 respectively and $\left[\begin{array}{l}n_{1}^{2} \\ n_{2}^{2}\end{array}\right]$ are the noise at time slot 2 on receive antenna 1, 2 respectively.Combining the equations at time slot 1 and 2,

$$
\begin{aligned}
{\left[\begin{array}{ll}
y_{1}^{1} & y_{1}^{2} \\
y_{2}^{1} & y_{2}^{2}
\end{array}\right] } & =\left[\begin{array}{ll}
h_{11} & h_{12} \\
h_{21} & h_{22}
\end{array}\right]\left[\begin{array}{cc}
a+b \theta & c+d \theta \\
\gamma(c+d \bar{\theta}) & a+b \bar{\theta}
\end{array}\right]+\left[\begin{array}{l}
n_{1}^{1} n_{1}^{2} \\
n_{2}^{1} n_{2}^{2}
\end{array}\right] \\
& =\mathrm{H} \square \mathrm{X}+\mathrm{N}
\end{aligned}
$$

When $\theta=\gamma=i$

$$
X=\left[\begin{array}{cc}
a+b \theta & c+d \theta \\
\gamma(c+d \bar{\theta}) & a+b \bar{\theta}
\end{array}\right]=\left[\begin{array}{cc}
a+b i & c+d i \\
i(c-d i) & a-b i
\end{array}\right]
$$

To solve for $X$, we know that we need to find the inverse of $H$.We know, for a general $\mathrm{m} \times \mathrm{n}$ matrix, thepseudo inverse operation is defined as $H^{+}=\left(H^{H} H\right)^{-1} H^{H}$.

The term,

$$
H^{H} H=\left[\begin{array}{ll}
\left|h_{11}\right|^{2}+\left|h_{21}\right|^{2} & h_{11}^{*} h_{12}+h_{21}^{*} h_{22} \\
h_{12}^{*} h_{11}+h_{22}^{*} h_{21} & \left|h_{12}\right|^{2}+\left|h_{22}\right|^{2}
\end{array}\right]
$$


From the equation above, we can see the diagonal element of the matrix is the received channel gain combination term. In the other side, $h_{11}^{*} h_{12}+h_{21}^{*} h_{22}$ is the correlation term between transmit antennas. If it is independent between transmit antenna 1 and transmit antenna 2,

$$
\begin{gathered}
H^{H} H=\left[\begin{array}{cc}
\left|h_{11}\right|^{2}+\left|h_{21}\right|^{2} & 0 \\
0 & \left|h_{12}\right|^{2}+\left|h_{22}\right|^{2}
\end{array}\right] \\
\left(H^{H} H\right)^{-1}=\left[\begin{array}{cc}
\frac{1}{\left|h_{11}\right|^{2}+\left|h_{21}\right|^{2}} & 0 \\
0 & \frac{1}{\left|h_{12}\right|^{2}+\left|h_{22}\right|^{2}}
\end{array}\right]
\end{gathered}
$$

\section{Watterson model}

The watterson model can be expressed in time domain as below equation.

$$
H(f, t)=\sum_{n=1}^{N} G_{n}(t) e^{-j 2 \pi f \tau_{n}}
$$

Where $G_{n}(t)$ denotes the channel gain for $n^{\text {th }}$ path, $\mathrm{N}$ denotes the number of propagation path.The power density function can be expressed as:

$$
\operatorname{si}(f)=\frac{R G_{i a}(0)}{\sqrt{2 \pi} \delta g_{i a}} \exp \left(-\frac{\left(f-f_{i a}\right)^{2}}{2 \delta^{2} g_{i a}}\right)+\frac{R G_{i b}(0)}{\sqrt{2 \pi} \delta g_{i b}} \exp \left(-\frac{\left(f-f_{i b}\right)^{2}}{2 \delta^{2} g_{i b}}\right)
$$

$\mathrm{G}_{\mathrm{ia}}(\mathrm{t})$ and $\mathrm{G}_{\mathrm{ib}}(\mathrm{t})$ are two independent complex (bivariate) -Gaussian stationary ergodic random processes, each with zero-mean values and independent quadrature components with equal rms values and identical spectrums.

It can be seen that the model involves three assumptions:

i)The Gaussian-scattering hypothesis, that each tap-gain function is a complex-Gaussian process;

ii)The independence hypothesis, that each tap-gain function is independent;

ii)The Gaussian-spectrum hypothesis, that each tap-gain'spectrum in general is the sum of two Gaussian functions of frequency.

\section{The Correlation Analysis}

$h_{11}^{*} h_{12}$ denotes the correlation coefficients between transmitter antennas for receiver antenna 1. $h_{21}^{*} h_{22}$ denotes the correlation coefficients between transmitter antennas for receiver antenna 2 . It is assumed that the transmission correlation for receiver antennas is the same. And the channel gain for one receiver antenna is a constant, i.e. 1.

We model the spatially correlated Rician MIMO channel as

$$
H=\sqrt{\frac{K}{K+1}} H_{\text {los }}+\sqrt{\frac{1}{K+1}} H_{\text {nlos }}
$$

Where $\mathrm{K}$ is Rician K-factor, and $H_{\text {los }}$ and $H_{\text {nlos }}$ are LOS and non-LOS(NLOS) components, respectively. We normalize the MIMO channel matrix such that $\varepsilon\left[\|H\|_{F}^{2}\right]=N_{r} N_{t}$. The NLOS channel matrix is given by

$$
H_{\text {nlos }}=R^{1 / 2} Z S^{1 / 2}
$$

Where $Z \in \square^{N_{r} \times N_{t}}$ contains independent complex i.i.d. Gaussian entries with zero mean and unit variance, and $\mathrm{S}$ and $\mathrm{R}$ denote the transmit and receive spatial correlation matrices, respectively, 
with eigenvalue decompositions.

$$
S=U_{s} \Lambda_{s} U_{s}^{\dagger}, R=U_{r} \Lambda_{r} U_{r}^{\dagger}
$$

For Golden Code with linear receivers, the MIMO channel is effectively decoupled into $\mathrm{Nt}$ parallel streams, for which the capacity is given by [6].

$$
C=\sum_{k=1}^{N_{t}} \varepsilon_{\gamma_{k}}\left[\log _{2}\left(1+\gamma_{k}\right)\right]
$$

Where $\gamma_{k}$ is the conditional postprocessing SNR for the kth stream. We consider minimum mean-square error (MMSE) and zero-forcing (ZF) linear receiver, for which $\gamma_{k}$ is given by

$$
\begin{gathered}
\gamma_{k}=\frac{1}{\left[\left(I_{N_{t}}+\frac{\gamma_{0}}{N_{t}} H^{H} H\right)^{-1}\right]_{k, k}}-1 \\
\gamma_{k}=\frac{\gamma_{0}}{N_{t}} \frac{1}{\left[\left(H^{H} H\right)^{-1}\right]_{k, k}}
\end{gathered}
$$

Respectively, where []$_{k, k}$ denotes the kth diagonal element.

To make our analysis mathematically tractable, we consider transmit correlated Watterson MIMO channel and ZF receiver. Note, however, that MMSE receiver slightly outperform ZF receivers at low to moderate SNRs. For the ZF case with transmit correlation, $\gamma_{k}$ has pdf which is correlated to the Watterson model.

$$
\begin{aligned}
& \gamma_{1}=\frac{\gamma_{0}}{N_{t}} \frac{\left(\left|h_{11}\right|^{2}+\left|h_{21}\right|^{2}\right)\left(\left|h_{12}\right|^{2}+\left|h_{22}\right|^{2}\right)-\left(h_{11}^{*} h_{12}+h_{21}^{*} h_{22}\right)\left(h_{12}^{*} h_{11}+h_{22}^{*} h_{21}\right)}{\left|h_{12}\right|^{2}+\left|h_{22}\right|^{2}} \\
& =\frac{\gamma_{0}}{N_{t}} \frac{\left(\left|h_{11}\right|^{2}+\left|h_{21}\right|^{2}\right)\left(\left|h_{12}\right|^{2}+\left|h_{22}\right|^{2}\right)-\left|h_{11}\right|^{2}\left|h_{12}\right|^{2}-\left|h_{21}\right|^{2}\left|h_{22}\right|^{2}-\left(h_{11}^{*} h_{12} h_{22}^{*} h_{21}+h_{12}^{*} h_{11} h_{21}^{*} h_{22}\right)}{\left|h_{12}\right|^{2}+\left|h_{22}\right|^{2}}
\end{aligned}
$$

The BER upper limit can be deduced from the bit error ratio equation

$$
p_{e}=2\left(1-\frac{1}{\sqrt{M}}\right) Q\left(\sqrt{\frac{3}{M-1}} \gamma\right)
$$

For $\left(h_{11}^{*} h_{12} h_{22}^{*} h_{21}+h_{12}^{*} h_{11} h_{21}^{*} h_{22}\right)$ terms, we can analysis the expectation analysis based on joint probability density function.

$$
E\left(h_{11}^{*} h_{12} h_{22}^{*} h_{21}+h_{12}^{*} h_{11} h_{21}^{*} h_{22}\right)=2 \Re\left(E\left(h_{11}^{*} h_{12} h_{22}^{*} h_{21}\right)\right)
$$

It is well known that the power density function is the fourier transformation of cross-correlation of random variables.

$$
\begin{aligned}
& E\left(h_{11}^{*} h_{12} h_{22}^{*} h_{21}\right)=f\left(P\left(h_{11} h_{12} h_{21} h_{22}\right)\right) \\
& =P\left(f\left(h_{11} h_{12} h_{21} h_{22}\right)\right) \\
& =\iiint \int s i_{11}(f) s i_{12}(v) s i_{21}(r) s i_{22}(t) d f d v d r d t
\end{aligned}
$$

Where $f(\bullet)$ denotes the fourier transformation. And it is a super- dimension integration, we cannot get the analytical solution.

\section{Simulation Results}

The Golden Code with different correlation coefficient by simulation using MATLAB in this 
paper. Channel simulation model using HF Watterson model, modulation using 4-QAM.Figure.2 show the relationship between BER and correlation coefficient.

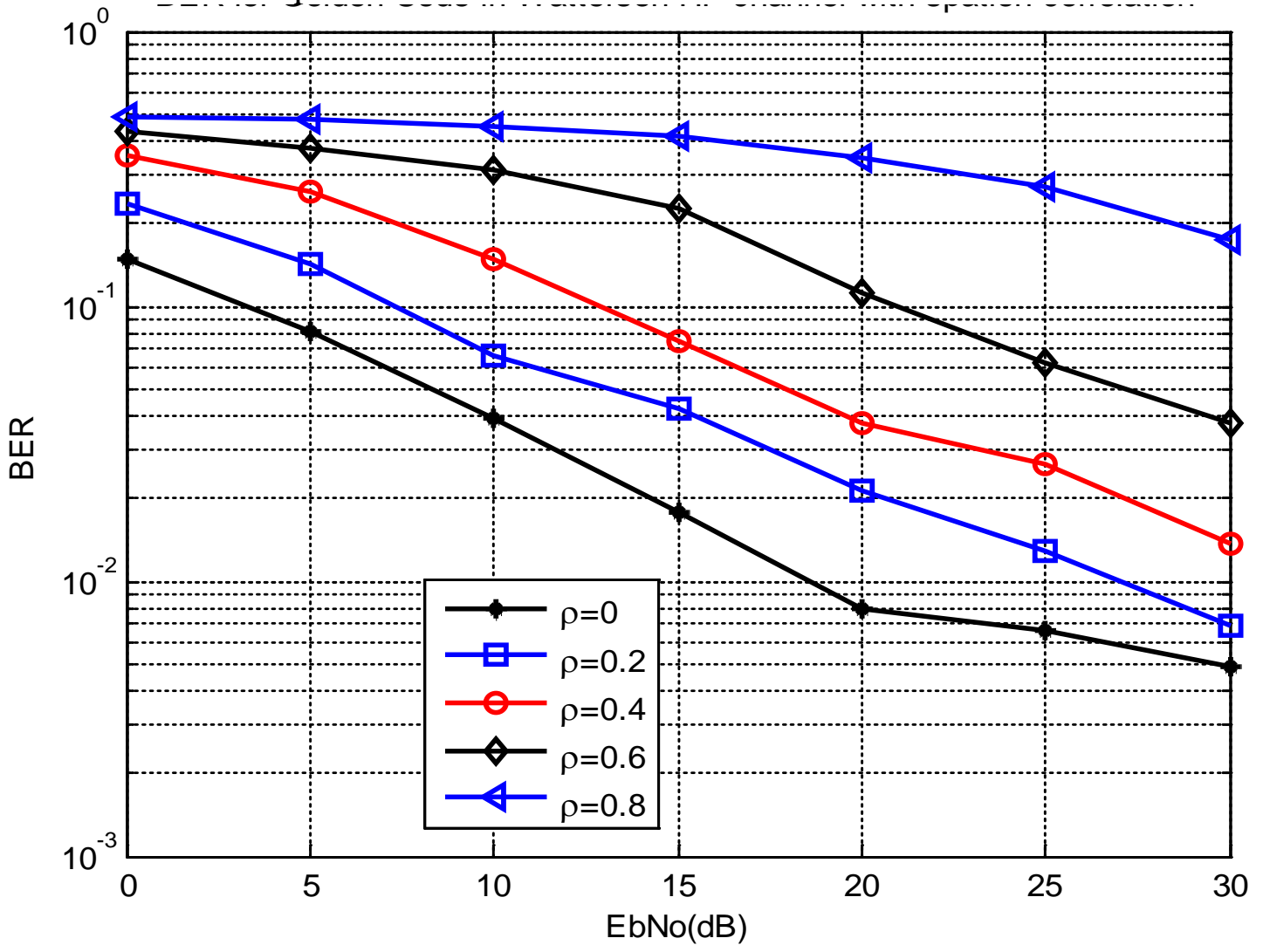

Fig2. BER for Golden Code in Watterson HF channel with spation correlation

It can be seen from the simulation Fig.2 that the impact of spatial correlation is obvious to Golden Code under the HF channel. When correlation coefficient is 0.2,performance of the system is decreased by $5 \mathrm{~dB}-7 \mathrm{~dB}$ compared to the independent channel. With the increase of the correlation coefficient, System performance degradation is still obviously.

\section{Conclusion}

This paper Analysis of Correlation on performance of Golden Code based on HF Watterson model. The results of simulation show that system performance degradation is obviously under the HF communication withspation correlation. So how to reduce the impact of spation correlation to the Golden Code is the focus of future research.

\section{Acknowledgements}

This research was financially supported by Theory and Technology of Collaborative MIMO communications(supported by the special fund of Chongqing key laboratory(CSTC))and National Natural Science Foundation of China (Grant No. 61271251).

\section{References}

[1] Xu, S., Zhang, H., Yang, H., \& Wang, H. New considerations for high frequency communications.In Communications, 2004 and the 5th International Symposium on Multi-Dimensional Mobile Communications Proceedings.The 2004 Joint Conference of the 10th Asia-Pacific Conference on (Vol. 1, pp. 444-447).IEEE.2004.

[2] Jie, Y., Yong, L., \& Na, W. The Study on L-STBC-OFDM in HF Communication System. In Optoelectronics and Image Processing (ICOIP), 2010 International Conference on (Vol. 2, pp. 
641-644). IEEE.2010.

[3] Uysal, M., \&Georghiades, C. N. Effect of spatial fading correlation on performance of space-time codes. Electronics Letters, 37(3), 181-183,2001.

[4] Gesbert, D., Bolcskei, H., Gore, D. A., \&Paulraj, A. J. Performance evaluation for scattering MIMO channel models. In Signals, Systems and Computers, 2000.Conference Record of the Thirty-Fourth Asilomar Conference on (Vol. 1, pp. 748-752).IEEE. October 2000.

[5]Belfiore, J. C., Rekaya, G., \&Viterbo, E. The Golden code: A 2 x 2 full-rate space-time code with nonvanishing determinants. IEEE Transactions on information theory, 51(4), 1432-1436,2005.

[6] Forenza, A., McKay, M. R., Pandharipande, A., Heath, R. W., \&Collings, I. B. (2007).Adaptive MIMO transmission for exploiting the capacity of spatially correlated channels. Vehicular Technology, IEEE Transactions on, 56(2), 619-630,2007. 